\title{
The Jost-Schroer Theorem for Zero-Mass Fields
}

\author{
K. Pohlmeyer \\ Department of Nuclear Physics \\ Received January 14, 1969
}

Weizmann Institute of Science, Rehovot, Israel

\begin{abstract}
We extend the Jost-Schroer theorem to zero-mass fields in one timedimension and arbitrarily many space-dimensions.
\end{abstract}

Recently, some work has been devoted to free zero-mass fields $[1,2]$. It might be useful to have a simple criterion for a field to be a free zeromass field. We shall give such a criterion for the sake of simplicity for a neutral scalar field, though similar criteria can readily be obtained in the more general case of fields transforming according to a finite dimensional representation of the Lorentz group.

Theorem (JosT and ScHROER [3]). If $\phi(x)$ is a hermitian scalar local field, relatively local to a set of fields for which the unique vacuum $\Omega$ is cyclic, and if

$$
(\Omega, \phi(x) \phi(y) \Omega)=\frac{1}{i} \Delta_{(n)}^{+}(x-y, 0)
$$

then $\phi(x)$ is a free zero-mass field.

First, we shall prove this theorem for $n$ space-dimensions with $n \geqq 2$. The case $n=1$ will be treated separately.

Proof. We define $j(x)=\left(\frac{\partial^{2}}{\partial x^{02}}-\sum_{i=1}^{n} \frac{\partial^{2}}{\partial x^{i 2}}\right) \phi(x)$. From the assumed structure of the 2 point function it follows that $j(x)$ annihilates the vacuum. We then apply the Johnson-Federbush theorem [4] and conlude that $j(x)=0$.

It remains to be shown that $[\phi(x), \phi(y)]$ is a $c$-number. Again because of the Johnson-Federbush theorem it is sufficient to prove

i.e.

$$
\left\{[\phi(x), \phi(y)]-\frac{1}{i} \Delta_{(n)}(x-y, 0)\right\} \Omega=0
$$

$$
\begin{aligned}
& \left(\Omega,\left[\phi\left(x_{1}\right), \phi\left(x_{2}\right)\right]\left[\phi\left(x_{3}\right), \phi\left(x_{4}\right)\right] \Omega\right) \\
& \quad=\left(\Omega,\left[\phi\left(x_{1}\right), \phi\left(x_{2}\right)\right] \Omega\right)\left(\Omega,\left[\phi\left(x_{3}\right), \phi\left(x_{4}\right)\right] \Omega\right) .
\end{aligned}
$$

* On leave of absence from II. Institut für Theoretische Physik der Universität, Hamburg, Germany. Work supported by ,Stiftung Volkswagenwerk“. 
Moreover, as a consequence of the positive definiteness condition, integration of

$\hat{W}\left(p_{1}, p_{2}, p_{3}\right)=$ Fourier-transform of

$$
\begin{aligned}
& \left\{W\left(x_{1}-x_{2}, \frac{x_{1}+x_{2}}{2}-\frac{x_{3}+x_{4}}{2}, x_{3}-x_{4}\right)\right. \\
= & \left.\left(\Omega,\left[\phi\left(x_{1}\right), \phi\left(x_{2}\right)\right]\left[\phi\left(x_{3}\right), \phi\left(x_{4}\right)\right] \Omega\right)\right\}
\end{aligned}
$$

over $p_{1}$ and $p_{3}$ with test functions $\in \mathscr{S}$ will give a measure in $p_{2}$. We have assumed that $\Omega$ is the only eigenstate belonging to the eigenvalue 0 of the energy momentum operator. Therefore, it will suffice to prove that the support of $\hat{W}\left(p_{1}, p_{2}, p_{3}\right)$ is concentrated in $p_{2}=0$. From the spectrum condition we know that

$$
\operatorname{supp} \hat{W}\left(p_{1}, p_{2}, p_{3}\right) \subset\left\{p_{1}, p_{2}, p_{3} / p_{2}^{2} \geqq 0, p_{20} \geqq 0\right\}
$$

i.e. $\hat{W}\left(p_{1}, p_{2}, p_{3}\right)=0$ unless $p_{2}$ lies in or on the forward cone.

$$
k \cdot l=k_{0} \cdot l_{0}-\sum_{i=1}^{n} k_{i} \cdot l_{i}, \quad k^{2}=k \cdot k, \quad \boldsymbol{k}=\left(k_{1}, \ldots, k_{n}\right) .
$$

In the first step, we show that

$$
\operatorname{supp} \hat{W}\left(p_{1}, p_{2}, p_{3}\right) \subset\left\{p_{1}, p_{2}, p_{3} / p_{2}^{2}=0, p_{20} \geqq 0\right\},
$$

i.e. $\hat{W}\left(p_{1}, p_{2}, p_{3}\right)=0$ unless $p_{2}$ lies on the forward cone.

From $j(x)=0$ it follows that

$$
\begin{aligned}
\operatorname{supp} \hat{W}\left(p_{1}, p_{2}, p_{3}\right) \subset\left\{p_{1}, p_{2}, p_{3} / p_{1} \cdot p_{2}\right. & =0, \\
p_{1}^{2}+\frac{p_{2}^{2}}{4} & =0, p_{2} \cdot p_{3}=0, \\
p_{3}^{2}+\frac{p_{2}^{2}}{4} & \left.=0, p_{2} \geqq 0, p_{20} \geqq 0\right\} .
\end{aligned}
$$

We now smear $\hat{W}\left(p_{1}, p_{\mathbf{2}}, p_{\mathbf{3}}\right)$ in $p_{\mathbf{2}}$ with a test function $\tilde{\psi} \in \mathscr{D}$ that has (compact) support in

$$
\check{V}_{+}=\left\{p_{2} / p_{20}>0, p_{2}^{2}>0\right\} \text { to obtain } \hat{W}_{\psi}\left(p_{1}, p_{3}\right) .
$$

Then we notice that $\operatorname{supp} \hat{W}_{\psi}\left(p_{1}, p_{3}\right)$ is compact. Consequently, $W_{\psi}\left(x_{1}-x_{2}, x_{3}-x_{4}\right)$ is an analytic function of its variables, vanishing because of locality for $\left(x_{1}-x_{2}\right)^{2}<0$ or $\left(x_{3}-x_{4}\right)^{2}<0$. Therefore

$$
W_{\psi}\left(x_{1}-x_{2}, x_{3}-x_{4}\right) \equiv 0 .
$$

This identity is true for all test function $\psi \in \mathscr{Z}=\tilde{\mathscr{D}}: \operatorname{supp} \tilde{\psi} \subset \stackrel{\circ}{V}_{+}$. 
That implies

$\operatorname{supp} \hat{W}\left(p_{1}, p_{2}, p_{3}\right)$

$$
\begin{aligned}
& \subset\left\{p_{1}, p_{2}, p_{3} / p_{2}^{2}=0, p_{20} \geqq 0, p_{1} \cdot p_{2}=p_{2} \cdot p_{3}=0, p_{1}^{2}=p_{3}^{2}=0\right\} \\
= & \left\{p_{1}, p_{2}, p_{3} / p_{2}=0, p_{1}^{2}=p_{3}^{2}=0\right\} \\
\cup & \left\{p_{1}, p_{2}, p_{3} / p_{2}^{2}=0, p_{2}^{0}>0, p_{1}=\lambda p_{2}, p_{3}=\mu p_{2},\right. \\
& -\infty<\lambda<+\infty,-\infty<\mu<+\infty\} .
\end{aligned}
$$

In the second and final step of this proof we show that indeed

$$
\operatorname{supp} \hat{W}\left(p_{1}, p_{2}, p_{3}\right) \subset\left\{p_{1}, p_{2}, p_{3} / p_{2}=0\right\} \text {. }
$$

For that, we choose a test function $\tilde{\psi} \in \mathscr{D}: \operatorname{supp} \tilde{\psi}\left(p_{2}\right)$ is concentrated around some arbitrary point $p_{2}$ on the forward cone with $p_{2}^{2}=0, p_{2}^{0}>0$, $\left\{p_{2}=0\right\} \notin \operatorname{supp} \tilde{\psi}\left(p_{2}\right)$. In addition, we take an arbitrary test function $f_{1} \in \mathscr{S}\left(R^{1}\right)$ and form

$$
\hat{W}_{f_{1}, \psi}\left(\boldsymbol{p}_{1}, p_{3}\right)=\int d p_{10} \int d p_{2} f_{1}\left(p_{10}\right) \tilde{\psi}\left(p_{2}\right) \hat{W}\left(p_{1}, p_{2}, p_{3}\right)
$$

$\hat{W}_{f_{1}, \psi}\left(\boldsymbol{p}_{1}, p_{3}\right)$ is a tempered distribution which, because of locality, after integration over $p_{3}$ with a test function $\in \mathscr{P}$ gives a $C^{\infty}$-function in $\boldsymbol{p}_{1}$. Therefore, the restriction to an arbitrary fixed vector $\boldsymbol{q}_{\mathbf{1}} \neq \mathbf{0} ; \boldsymbol{p}_{\mathbf{1}}=\boldsymbol{q}_{\mathbf{1}}$ exists [5] and defines a tempered distribution $W_{f_{1}, \psi}^{\boldsymbol{q}_{1}}\left(p_{3}\right)$ in $p_{3}$.

$$
\operatorname{supp} W_{f_{1}, \psi}^{\boldsymbol{q}_{1}}\left(p_{3}\right) \subset\left\{p_{3} / \boldsymbol{p}_{3}=\varrho \boldsymbol{q}_{1}, p_{3}^{2}=0,-\infty<\varrho<+\infty\right\} .
$$

The Fourier-transform of $W_{f_{1}, \psi}^{\boldsymbol{q}_{1}}\left(p_{3}\right)$, i.e. $W_{f_{1}, \psi}^{\boldsymbol{q}_{1}}\left(x_{3}-x_{4}\right)$, vanishes for $\left(x_{3}-x_{4}\right)^{2}<0$. Again we choose an arbitrary test function $f_{3} \in \mathscr{S}\left(R^{1}\right)$ and form $W_{f_{1}, \psi, f_{3}}^{\boldsymbol{q}_{1}}\left(\boldsymbol{p}_{3}\right)=\int d p_{30} \tilde{f}_{3}\left(p_{30}\right) \hat{W}_{f_{1}, \psi}^{\boldsymbol{q}_{1}}\left(p_{3}\right)$, which is a $C^{\infty}$-function in $\boldsymbol{p}_{3}$. However, since the support of $\hat{W}_{f_{1}, \psi, f_{3}}^{\boldsymbol{q}_{1}}\left(\boldsymbol{p}_{3}\right)$ is concentrated on the line $\boldsymbol{p}_{3}=\varrho \boldsymbol{q}_{1}$, we run into a contradiction (for $n \geqq 2$ only) unless

$$
\hat{W}_{f_{1}, \psi, f_{s}}^{\boldsymbol{q}_{1}}\left(\boldsymbol{p}_{3}\right) \equiv 0 \text {. }
$$

Apart from the constraint $\boldsymbol{q}_{1} \neq 0$, the vector $\boldsymbol{q}_{\mathbf{1}}$ is arbitrary. Thus we obtain

$\hat{W}_{f_{1}, \psi, f_{3}}\left(\boldsymbol{p}_{1}, \boldsymbol{p}_{3}\right)=\int d p_{10} \int d p_{2} \int d p_{30} \tilde{f}_{1}\left(p_{10}\right) \tilde{\psi}\left(p_{2}\right) \tilde{f}_{3}\left(p_{30}\right) \hat{W}\left(p_{1}, p_{2}, p_{3}\right) \equiv 0$ in $\left\{\boldsymbol{p}_{1}, \boldsymbol{p}_{3} / \boldsymbol{p}_{1} \neq 0\right\}$.

From the continuity of $\hat{W}_{f_{1}, \psi, f_{3}}\left(\boldsymbol{p}_{1}, \boldsymbol{p}_{3}\right)$ in both variables (a consequence of locality) we infer

$$
\hat{W}_{f_{1}, \psi, f_{3}}\left(\boldsymbol{p}_{1}, \boldsymbol{p}_{3}\right) \equiv 0 \text { for all } \boldsymbol{p}_{1}, \boldsymbol{p}_{3},
$$

and recalling that $f_{1}$ and $f_{3}$ were arbitrary test functions $\in \mathscr{S}\left(R^{1}\right)$, we conclude that i.e.

$$
\hat{W}_{\psi}\left(p_{1}, p_{3}\right) \equiv 0 \quad \text { for all } \quad \psi \in \tilde{\mathscr{D}} \quad \text { with } \quad\left\{p_{2}=0\right\} \notin \operatorname{supp} \tilde{\psi}\left(p_{2}\right)
$$

$$
\operatorname{supp} \hat{W}\left(p_{1}, p_{2}, p_{3}\right) \subset\left\{p_{1}, p_{2}, p_{3} / p_{2}=0, p_{1}^{2}=p_{3}^{2}=0\right\} . \quad \text { q.e.d. }
$$


In the second part of this contribution we shall prove the theorem for one time and one space dimension. This case is of some interest for field theoretic models. Many of those that are explicitly soluable are models in one time and one space dimension. It is well known [6] that in two-dimensional space-time no free scalar field of mass zero exists if one imposes on it the usual requirements of quantum field theory, especially the positive definiteness condition. Thus care is needed here.

To begin with, we shall prove the following lemma.

Lemma. If $j_{v}(x)$ is a hermitian local vector field in one time and one space dimension, relatively local to a set of fields for which the unique vacuum $\Omega$ is cyclic, and if

$$
\left(\Omega, j_{\mu}(x) j_{\nu}(y) \Omega\right)=\frac{1}{i} \frac{\partial^{2}}{\partial x^{\mu} \partial y^{v}} \Delta_{(1)}^{+}(x-y, 0)
$$

then $j_{v}(x)$ is a free zero-mass vector field with

$$
\operatorname{div} j(x)=0, \quad \operatorname{curl} j(x)=0 .
$$

Proof. From the assumed structure of the 2 point function we obtain at once that i.e.

$$
\|\operatorname{div} j(x) \Omega\|^{2}=0 \quad \text { and } \quad\|\operatorname{curl} j(x) \Omega\|^{2}=0
$$

$$
\operatorname{div} j(x) \Omega=0 \quad \text { and } \quad \operatorname{curl} j(x) \Omega=0 .
$$

The Johnson-Federbush theorem implies that

$$
\operatorname{div} j(x)=0 \text { and } \operatorname{curl} j(x)=0,
$$

and that gives immediately

We define

$$
\left(\frac{\partial^{2}}{\partial x^{02}}-\frac{\partial^{2}}{\partial x^{12}}\right) j_{v}(x)=0
$$

$$
\begin{aligned}
& j_{+}(x)=j_{0}(x)+j_{1}(x), \\
& j_{-}(x)=j_{0}(x)-j_{1}(x) .
\end{aligned}
$$

The proof of the lemma will be established if we can show that the commutators $\left[j_{\sigma_{1}}(x), j_{\sigma_{2}}(y)\right]$ are $c$-numbers $\left(\sigma_{i}=+\right.$ or $\left.-, i=1,2\right)$. Once more, because of the Johnson-Federbush theorem it suffices to prove that

$$
\begin{aligned}
& \left(\Omega,\left[j_{\sigma_{1}}\left(x_{1}\right), j_{\sigma_{2}}\left(x_{2}\right)\right]\left[j_{\sigma_{2}}\left(x_{3}\right), j_{\sigma_{1}}\left(x_{4}\right)\right] \Omega\right) \\
& \quad=\left(\Omega,\left[j_{\sigma_{1}}\left(x_{1}\right), j_{\sigma_{2}}\left(x_{2}\right)\right] \Omega\right)\left(\Omega,\left[j_{\sigma_{2}}\left(x_{3}\right), j_{\sigma_{1}}\left(x_{4}\right)\right] \Omega\right)
\end{aligned}
$$

We introduce new coordinates $x^{+}$and $x^{-}$

$$
x^{+}=x^{0}+x^{1}, \quad x^{-}=x^{0}-x^{1} .
$$

In these new coordinates $\operatorname{div} j(x)=0$ and $\operatorname{curl} j(x)=0$ read

$$
\frac{\partial}{\partial x^{-}} j_{+}\left(x^{+}, x^{-}\right)=0 \text { and } \quad \frac{\partial}{\partial x^{+}} j_{-}\left(x^{+}, x^{-}\right)=0 .
$$


Now we consider

and

$$
\left(\Omega,\left[j_{\sigma_{1}}\left(x_{1}\right), j_{\sigma_{2}}\left(x_{2}\right)\right] \Omega\right)
$$

$$
\left(\Omega,\left[j_{\sigma_{1}}\left(x_{1}\right), j_{\sigma_{2}}\left(x_{2}\right)\right]\left[j_{\sigma_{2}}\left(x_{3}\right), j_{\sigma_{1}}\left(x_{4}\right)\right] \Omega\right) .
$$

The differential equations imply that these distributions depend only on $x_{1}^{\sigma_{1}}, x_{2}^{\sigma_{2}}$ and $x_{1}^{\sigma_{1}}, x_{2}^{\sigma_{2}}, x_{3}^{\sigma_{2}}, x_{4}^{\sigma_{1}}$ rsp., i.e.

and

$$
\left(\Omega,\left[j_{\sigma_{1}}\left(x_{1}\right), j_{\sigma_{2}}\left(x_{2}\right)\right] \Omega\right)=W_{\sigma_{1} \sigma_{2}}\left(x_{1}^{\sigma_{1}}, x_{2}^{\sigma_{2}}\right)
$$

$$
\left(\Omega,\left[j_{\sigma_{1}}\left(x_{1}\right), j_{\sigma_{2}}\left(x_{2}\right)\right]\left[j_{\sigma_{2}}\left(x_{3}\right), j_{\sigma_{1}}\left(x_{4}\right)\right] \Omega\right)=W_{\sigma_{1} \sigma_{2} \sigma_{2} \sigma_{1}}\left(x_{1}^{\sigma_{1}}, x_{2}^{\sigma_{2}}, x_{3}^{\sigma_{2}}, x_{4}^{\sigma_{1}}\right) .
$$

It follows from translation invariance that for all real $a^{\sigma_{1}}, a^{\sigma_{2}}$

and

$$
W_{\sigma_{1} \sigma_{2}}\left(x_{1}^{\sigma_{1}}, x_{2}^{\sigma_{2}}\right)=W_{\sigma_{1} \sigma_{2}}\left(x_{1}^{\sigma_{1}}+a^{\sigma_{1}}, x_{2}^{\sigma_{2}}+a^{\sigma_{2}}\right)
$$

$$
\begin{aligned}
& W_{\sigma_{1} \sigma_{2} \sigma_{2} \sigma_{1}}\left(x_{1}^{\sigma_{1}}, x_{2}^{\sigma_{2}}, x_{3}^{\sigma_{2}}, x_{4}^{\sigma_{1}}\right) \\
& =W_{\sigma_{1} \sigma_{2} \sigma_{2} \sigma_{1}}\left(x_{1}^{\sigma_{1}}+a^{\sigma_{1}}, x_{2}^{\sigma_{2}}+a^{\sigma_{2}}, x_{3}^{\sigma_{2}}+a^{\sigma_{2}}, x_{4}^{\sigma_{1}}+a^{\sigma_{1}}\right) .
\end{aligned}
$$

For $\sigma_{1} \neq \sigma_{2}$ this means that

is a constant and

$$
\left(\Omega,\left[j_{\sigma_{1}}\left(x_{1}\right), j_{\sigma_{2}}\left(x_{2}\right)\right] \Omega\right)
$$

$$
\left(\Omega,\left[j_{\sigma_{1}}\left(x_{1}\right), j_{\sigma_{2}}\left(x_{2}\right)\right]\left[j_{\sigma_{2}}\left(x_{3}\right), j_{\sigma_{1}}\left(x_{4}\right)\right] \Omega\right)
$$

depends only upon $x_{1}^{\sigma_{1}}-x_{4}^{\sigma_{1}}$ and $x_{2}^{\sigma_{2}}-x_{3}^{\sigma_{2}}$. The locality condition then requires both

$$
\left(\Omega,\left[j_{\sigma_{1}}\left(x_{1}\right), j_{\sigma_{2}}\left(x_{2}\right)\right] \Omega\right) \text { and }\left(\Omega,\left[j_{\sigma_{1}}\left(x_{1}\right), j_{\sigma_{2}}\left(x_{2}\right)\right]\left[j_{\sigma_{2}}\left(x_{3}\right), j_{\sigma_{1}}\left(x_{4}\right)\right] \Omega\right)
$$

to be identical zero. Evidently, we have for $\sigma_{1} \neq \sigma_{2}$

$$
\begin{aligned}
& \left(\Omega,\left[j_{\sigma_{1}}\left(x_{1}\right), j_{\sigma_{2}}\left(x_{2}\right)\right]\left[j_{\sigma_{2}}\left(x_{3}\right), j_{\sigma_{1}}\left(x_{4}\right)\right] \Omega\right) \\
& =\left(\Omega,\left[j_{\sigma_{1}}\left(x_{1}\right), j_{\sigma_{2}}\left(x_{2}\right)\right] \Omega\right)\left(\Omega,\left[j_{\sigma_{2}}\left(x_{3}\right), j_{\sigma_{1}}\left(x_{4}\right)\right] \Omega\right) .
\end{aligned}
$$

The argument that will lead us to the corresponding relation for the remaining case $\sigma_{1}=\sigma_{2}=\sigma$ is more involved. From the foregoing discussion we know that $\left(\Omega,\left[j_{\sigma}\left(x_{1}\right), j_{\sigma}\left(x_{2}\right)\right] \Omega\right)$ and $\left(\Omega,\left[j_{\sigma}\left(x_{1}\right), j_{\sigma}\left(x_{2}\right)\right]\left[j_{\sigma}\left(x_{3}\right)\right.\right.$, $\left.j_{\sigma}\left(x_{4}\right)\right] \Omega$ ) depend only upon the variables $x_{1}^{\sigma}-x_{2}^{\sigma}$ and $x_{1}^{\sigma}-x_{2}^{\sigma}, \frac{x_{1}^{\sigma}+x_{2}^{\sigma}}{2}$ $-\frac{x_{3}^{\sigma}+x_{4}^{\sigma}}{2}, x_{3}^{\sigma}-x_{4}^{\sigma}$ rsp. It follows from locality that the supports of $\left(\Omega,\left[j_{\sigma}\left(x_{1}\right), j_{\sigma}\left(x_{2}\right)\right] \Omega\right)$ and $\left(\Omega,\left[j_{\sigma}\left(x_{1}\right), j_{\sigma}\left(x_{2}\right)\right]\left[j_{\sigma}\left(x_{3}\right), j_{\sigma}\left(x_{4}\right)\right] \Omega\right)$ are concentrated in $x_{1}^{\sigma}-x_{2}^{\sigma}=0$ and $x_{1}^{\sigma}-x_{2}^{\sigma}=0=x_{3}^{\sigma}-x_{4}^{\sigma}$ rsp. Thus, by invoking the temperedness condition, we obtain the following representations:

$$
\begin{gathered}
\left(\Omega,\left[j_{\sigma}\left(x_{1}\right), j_{\sigma}\left(x_{2}\right)\right] \Omega\right)=\sum_{\lambda=0}^{L_{\sigma}} c_{\sigma}^{\lambda} \delta^{(\lambda)}\left(x_{1}^{\sigma}-x_{2}^{\sigma}\right), \\
\left(\Omega,\left[j_{\sigma}\left(x_{1}\right), j_{\sigma}\left(x_{2}\right)\right]\left[j_{\sigma}\left(x_{3}\right), j_{\sigma}\left(x_{4}\right)\right] \Omega\right)=\sum_{\mu=0}^{M_{\sigma}} \sum_{\mu^{\prime}=0}^{M_{\sigma}^{\prime}} \delta^{(\mu)}\left(x_{1}^{\sigma}-x_{2}^{\sigma}\right) \\
\omega_{\sigma}^{\mu \mu^{\prime}}\left(\frac{x_{1}^{\sigma}+x_{2}^{\sigma}}{2}-\frac{x_{3}^{\sigma}+x_{4}^{\sigma}}{2}\right) \delta^{\left(\mu^{\prime}\right)}\left(x_{3}^{\sigma}-x_{4}^{\sigma}\right) .
\end{gathered}
$$


Here, $L_{\sigma}, M_{\sigma}$ and $M_{\sigma}^{\prime}$ are some fixed positive integers, the $c_{\sigma}^{\lambda}$ are complex numbers and the $\omega_{\sigma}^{\mu \mu^{\prime}}\left(\frac{x_{1}^{\sigma}+x_{2}^{\sigma}}{2}-\frac{x_{3}^{\sigma}+x_{4}^{\sigma}}{2}\right)$ are tempered distributions whose Fourier transforms $\tilde{\omega}_{\sigma}^{\mu \mu^{\prime}}\left(p_{\sigma}\right)$ are polynomially bounded complex measures which vanish unless $p_{\sigma} \geqq 0$. This last assertion follows from the temperedness, positive definiteness and spectrum conditions.

Next, we make use of the Lorentz covariance which yields

$$
\begin{aligned}
& \sum_{\lambda=0}^{L_{\sigma}} c_{\sigma}^{\lambda} \delta^{(\lambda)}\left(x_{1}^{\sigma}-x_{2}^{\sigma}\right)=\alpha^{2} \sum_{\lambda=0}^{L_{\sigma}} c_{\sigma}^{\lambda} \delta^{(\lambda)}\left(\alpha\left\{x_{1}^{\sigma}-x_{2}^{\sigma}\right\}\right), \\
& \sum_{\mu=0}^{M_{\sigma}^{\sigma}} \sum_{\mu^{\prime}=0}^{M_{\sigma}^{\prime}} \delta^{(\mu)}\left(x_{1}^{\sigma}-x_{2}^{\sigma}\right) \omega_{\sigma}^{\mu \mu^{\prime}}\left(\frac{x_{1}^{\sigma}+x_{2}^{\sigma}}{2}-\frac{x_{3}^{\sigma}+x_{4}^{\sigma}}{2}\right) \delta^{\left(\mu^{\prime}\right)}\left(x_{3}^{\sigma}-x_{4}^{\sigma}\right) \\
& =\alpha^{4} \sum_{\mu=0}^{M_{\sigma}} \sum_{\mu^{\prime}=0}^{M_{\sigma}^{\prime}} \delta^{(\mu)}\left(\alpha\left\{x_{1}^{\sigma}-x_{2}^{\sigma}\right\}\right) \omega_{\sigma}^{\mu \mu^{\prime}}\left(\alpha\left\{\frac{x_{1}^{\sigma}+x_{2}^{\sigma}}{2}-\frac{x_{3}^{\sigma}+x_{4}^{\sigma}}{2}\right\}\right) \\
& \cdot \delta^{\left(\mu^{\prime}\right)}\left(\alpha\left\{x_{3}^{\sigma}-x_{4}^{\sigma}\right\}\right)
\end{aligned}
$$

for all positive $\alpha$. These conditions imply that

$$
c_{\sigma}^{\lambda} \delta^{(\lambda)}\left(x_{1}^{\sigma}-x_{2}^{\sigma}\right)=\alpha^{2} c_{\sigma}^{\lambda} \delta^{(\lambda)}\left(\alpha\left\{x_{1}^{\sigma}-x_{2}^{\alpha}\right\}\right)
$$

for all positive $\alpha, 0 \leqq \lambda \leqq L_{\sigma}$ and

$$
\begin{aligned}
\delta^{(\mu)}\left(x_{1}^{\sigma}-x_{2}^{\sigma}\right) \omega_{\sigma}^{\mu \mu^{\prime}}\left(\frac{x_{1}^{\sigma}+x_{2}^{\sigma}}{2}-\frac{x_{3}^{\sigma}+x_{4}^{\sigma}}{2}\right) \delta^{\left(\mu^{\prime}\right)}\left(x_{3}^{\sigma}-x_{4}^{\sigma}\right) \\
\quad=\alpha^{4} \delta^{(\mu)}\left(\alpha\left\{x_{1}^{\sigma}-x_{2}^{\sigma}\right\}\right) \omega_{\sigma}^{\mu \mu^{\prime}}\left(\alpha\left\{\frac{x_{1}^{\sigma}+x_{2}^{\sigma}}{2}-\frac{\left.x_{3}^{\sigma}+x_{4}^{\sigma}\right\}}{2}\right\}\right) \delta^{\left(\mu^{\prime}\right)}\left(\alpha\left\{x_{3}^{\sigma}-x_{4}^{\sigma}\right\}\right)
\end{aligned}
$$

for all positive $\alpha, 0 \leqq \mu \leqq M_{\sigma}, 0 \leqq \mu^{\prime} \leqq M_{\sigma}^{\prime}$, i.e.

$$
c_{\sigma}^{\lambda}=0 \text { for } \lambda \neq 1
$$

and

$$
\int d p \tilde{\psi}(-p) \tilde{\omega}_{\sigma}^{\mu \mu^{\prime}}(p)=\frac{\alpha^{2}}{\alpha^{\mu+\mu^{\prime}}} \int d p \tilde{\psi}(-\alpha p) \tilde{\omega}_{\sigma}^{\mu \mu^{\prime}}(p)
$$

for all test functions $\tilde{\psi} \in \mathscr{S}$ and for all positive $\alpha, 0 \leqq \mu \leqq M_{\sigma}$, $0 \leqq \mu^{\prime} \leqq M_{\sigma}^{\prime}$. This homogeneity leads us to the relation

$$
\left(2-\mu-\mu^{\prime}\right) \int d p \tilde{\psi}(-p) \tilde{\omega}_{\sigma}^{\mu \mu^{\prime}}(p)=\int d p \tilde{\psi}^{\prime}(-p) p \tilde{\omega}_{\sigma}^{\mu \mu^{\prime}}(p) .
$$

In the case: $\mu+\mu^{\prime} \geqq 2$, with the particular choice

$$
\tilde{\psi}(p)=p^{\mu+\mu^{\prime}-2} \widetilde{\Phi}(p)
$$

where $\widetilde{\Phi}(p)$ is an arbitrary test function $\in \mathscr{S}$, this relation becomes

$$
\int d p(-p)^{\mu+\mu^{\prime}-1} \widetilde{\Phi}^{\prime}(-p) \tilde{\omega}_{\sigma}^{\mu \mu^{\prime}}(p)=0 .
$$

Now we exploit the fact that the $\tilde{\omega}_{\sigma}^{\mu \eta^{\prime}}(p)$ are complex measures with contributions only from the points $p \geqq 0$ and conclude that

$$
\tilde{\omega}_{\sigma}^{\mu \mu^{\prime}}(p)=c_{\sigma}^{\mu \mu^{\prime}} \delta(p) \text { for } \mu+\mu^{\prime} \geqq 2 .
$$


Here the $c_{\sigma}^{\mu \mu^{\prime}}$ are complex constants. It follows from the homogeneity that

$$
c_{\sigma}^{\mu \mu^{\prime}}=0 \text { unless } \mu+\mu^{\prime}=2 .
$$

In the cases $\mu+\mu^{\prime}=1$ and $\mu=\mu^{\prime}=0$ we conclude that the $\tilde{\omega}_{\sigma}^{\mu \mu^{\prime}}(p)$ 's are constant and linearly increasing rsp., i.e.

and

$$
\tilde{\omega}_{\boldsymbol{\sigma}}^{\mu \mu^{\prime}}(p)=c_{\sigma}^{\mu \mu^{\prime}} \Theta(p), \quad \mu+\mu^{\prime}=1
$$

$$
\tilde{\omega}_{\sigma}^{00}(p)=c_{\sigma}^{00} p \Theta(p) .
$$

However, $\left(\Omega,\left[j_{\sigma}\left(x_{1}\right), j_{\sigma}\left(x_{2}\right)\right]\left[j_{\sigma}\left(x_{3}\right), j_{\sigma}\left(x_{4}\right)\right] \Omega\right)$ is antisymmetric under the interchange of $x_{1}$ and $x_{2}$, or of $x_{3}$ and $x_{4}$. Thus, $c_{\sigma}^{\mu \mu^{\prime}}=0$ unless $\mu=\mu^{\prime}=1$, and we are left with

$$
\begin{aligned}
\left(\Omega,\left[j_{\sigma}\left(x_{1}\right), j_{\sigma}\left(x_{2}\right)\right] \Omega\right) & =c_{\sigma}^{1} \delta^{(1)}\left(x_{1}^{\sigma}-x_{2}^{\sigma}\right) \\
\left(\Omega,\left[j_{\sigma}\left(x_{1}\right), j_{\sigma}\left(x_{2}\right)\right]\left[j_{\sigma}\left(x_{3}\right), j_{\sigma}\left(x_{4}\right)\right] \Omega\right) & =\frac{c_{\sigma}^{11}}{\sqrt{2 \pi}} \delta^{(1)}\left(x_{1}^{\sigma}-x_{2}^{\sigma}\right) \delta^{(1)}\left(x_{3}^{\sigma}-x_{4}^{\sigma}\right) .
\end{aligned}
$$

From the hermiticity and the positive definiteness condition, it follow that $c_{\sigma}^{1}$ is purely imaginary and that $c_{\sigma}^{11}$ is real and non-positive. From the assumed uniqueness of the vacuum we infer that $\frac{c_{\sigma}^{11}}{\sqrt{2 \pi}}=\left(c_{\sigma}^{1}\right)^{2}$ and we end up with $\left(\Omega,\left[j_{\sigma}\left(x_{1}\right), j_{\sigma}\left(x_{2}\right)\right]\left[j_{\sigma}\left(x_{3}\right), j_{\sigma}\left(x_{4}\right)\right] \Omega\right)$

$$
=\left(\Omega,\left[j_{\sigma}\left(x_{1}\right), j_{\sigma}\left(x_{2}\right)\right] \Omega\right) \cdot\left(\Omega,\left[j_{\sigma}\left(x_{3}\right), j_{\sigma}\left(x_{4}\right)\right] \Omega\right) . \quad \text { q.e.d. }
$$

In one time and one space dimension we can no longer impose the positive definiteness condition upon the hermitian local scalar field $\phi(x)$ because

with

$$
\Delta_{(1)}^{+}(x-y, 0)=\frac{1}{2 \pi} \int d \omega(p) e^{-i p(x-y)}
$$

$$
d \omega(p)=\left\{\frac{1}{\left(p_{0}-p_{1}\right)_{+}} \delta\left(p_{0}+p_{1}\right)+\frac{1}{\left(p_{0}+p_{1}\right)_{+}} \delta\left(p_{0}+p_{1}\right)+b \delta\left(p_{0}\right) \delta\left(p_{1}\right)\right\}
$$

is not a positive measure [6]. We rather impose the positive definiteness condition upon the derivatives of $\phi(x)$. In order to prove the Jost-Schroer theorem also in this case we only need to make sure that the commutator $[\phi(x), \phi(y)]$ is a $c$-number. As we saw it is only at this point that our general argument fails to be conclusive for two dimensional space-time.

We observe that the vector field $\partial_{\nu} \phi(x)$ satisfies the assumptions of the lemma from which then we conclude that for all test functions $f, g \in \stackrel{\circ}{\mathscr{S}}=\left\{h / h \in \mathscr{S}, \int d x h(x)=0\right\}[\phi(f), \phi(g)]$ is a $c$-number. We denote by $\mathscr{D}$ the set of all test functions $\in \stackrel{\circ}{\mathscr{S}}$ with compact support.

Now we take test functions $f \in \stackrel{\circ}{\mathscr{D}}$ and $g \in \mathscr{S}$. From the locality it follows that $[\phi(f), \phi(g)]=[\phi(f), \phi(\stackrel{\circ}{)})]$ is a $c$-number, where

$$
\stackrel{\circ}{g}(x)=g(x)-\chi^{f}(x) \int d x g(x) \in \stackrel{\circ}{\mathscr{S}}
$$


with $\chi^{f} \in \mathscr{D}, \int d x \chi^{f}(x)=1$ and supp $\chi^{f}$ space-like to supp $f$. We apply this argument once more and infer that $[\phi(f), \phi(g)]$ is a $c$-number for all test functions $f, g \in \mathscr{D}$. Finally, by appealing to continuity we find that $[\phi(f), \phi(g)]$ is a $c$-number for all test functions $f, g \in \mathscr{S}$. q.e.d.

It is quite remarkable that once a hermitian scalar local field has the 2 point function

$$
(\Omega, \phi(x) \phi(y) \Omega)=\frac{1}{i} \Delta_{(n)}^{+}(x-y, m)
$$

all higher order Wightman functions are fixed for $m>0, n \geqq 1$ and $m=0, n \geqq 2$. (For $m=0, n=1$ all higher order truncated Wightman functions are trivial in the sense that they do not depend on their arguments. The assertions concerning the case $m=0$ are consequences of our theorem and lemma.) In general, that need not be so. There are counter examples in the class of Wick polynomials where not even the 2 and 3 point functions fix all the remaining Wightman functions.

Acknowledgement. It is a pleasure to thank Professor A. DE-SHalit and Professor I. TALMI for the kind hospitality extended to me at the Department of Nuclear Physics at The Weizmann Institute of Science, Israel, and to thank the Foundation Volkswagenwerk for its generous support. Thanks are also due to Mr. M. ScHeUNERT, II. Institut für Experimentalphysik der Universität Hamburg, Germany, for helpful discussions.

\title{
References
}

1. Bender, C. M.: Phys. Rev. 168, 1809 (1968).

2. Frishman, Y., and C. Itzykson: SLAC-PUB-498, August 1968 (TH).

3. Jost, R.: Properties of Wightman functions. In: Lectures on field theory and the many-body problem, E. R. Cataniello (ed.). New York: Academic Press 1961.

4. Federbush, P. G., and K. A. Johnson: Phys. Rev. 120, 1926 (1960).

5. Gelfand, I. M., u. G. E. Schilow: Verallgemeinerte Funktionen (Distributionen) II. Berlin: VEB Deutscher Verlag der Wissenschaften 1962.

6. Wightman, A. S.: Introduction to some aspects of the relativistic dynamics of quantized fields. In: Cargèse lectures in theoretical physics 1964. New YorkLondon-Paris: Gordon and Breach 1964.

\author{
K. Pohlmeyer \\ Department of Nuclear Physics \\ Weizmann Institute of Science \\ Rehovot, Israel
}

